\title{
Spot Forming Units Per Million Peripheral Blood Mononuclear Cells
}

National Cancer Institute

\section{Source}

National Cancer Institute. Spot Forming Units Per Million Peripheral Blood Mononuclear

Cells. NCl Thesaurus. Code C120850.

A unit of measure equal to the spot forming units per million cells. 